\title{
Genetic predisposition to salt-sensitive normotension and its effects on salt taste perception and intake
}

\author{
Leta Pilic* and Yiannis Mavrommatis \\ School of Sport, Health and Applied Science, St Mary's University Twickenham, Waldegrave Road, Strawberry Hill, \\ Twickenham TW1 4SX, UK
}

(Submitted 3 November 2017 - Final revision received 28 June 2018 - Accepted 2 July 2018 - First published online 14 August 2018)

\section{Abstract}

Salt sensitivity is an independent CVD and mortality risk factor, which is present in both hypertensive and normotensive populations. It is genetically determined and it may affect the relationship between salt taste perception and salt intake. The aim of this study was to explore the genetic predisposition to salt sensitivity in a young and a middle-aged adult population and its effects on salt taste perception and salt intake. The effects of Na loading on blood pressure (BP) were investigated in twenty normotensive subjects and salt sensitivity defined as the change in BP after $7 \mathrm{~d}$ of low-Na $(51.3 \mathrm{mmol} \mathrm{Na} / \mathrm{d})$ and $7 \mathrm{~d}$ of high-Na diet $(307.8 \mathrm{mmol} \mathrm{Na} / \mathrm{d})$. Salt taste perception was identified using the British Standards Institution sensory analysis method (BS ISO 3972:2011). Salt intake was assessed with a validated FFQ. DNA was genotyped for SNP in the SLC4A5, SCNN1B and TRPV1 genes. The subjects with AA genotype of the SLC4A5 rs7571842 exhibited the highest increase in BP ( $\Delta$ systolic $\mathrm{BP}=7.75 \mathrm{mmHg}, P=0.002, d=2.4 ; \Delta$ diastolic $\mathrm{BP}=6.25 \mathrm{mmHg}, P=0.044, d=1.3 ; \Delta$ mean arterial pressure $=6.5 \mathrm{mmHg}$, $P=0.014, d=1.7)$. The SLC4A5 rs10177833 was associated with salt intake $(P=0.037)$, and there was an association between salt taste perception and salt sensitivity $\left(r_{\mathrm{s}} 0.551, P=0.041\right)$. In conclusion, there is a genetic predisposition to salt sensitivity and it is associated with salt taste perception. The association between salt taste perception and discretionary salt use suggests that preference for salty taste may be a driver of salt intake in a healthy population and warrants further investigation.

Key words: Blood pressure: Genetics: Salt sensitivity: Salt intake: Taste

Hypertension is a major cause of CVD and overall mortality ${ }^{(1)}$. High dietary $\mathrm{Na}$ intake is a major risk factor for hypertension ${ }^{(2,3)}$ estimated to be responsible for one in ten deaths from CVD events $^{(4)}$. In 2010, the estimated mean global Na consumption was $3.95 \mathrm{~g} / \mathrm{d}$, with regional mean levels ranging from 2.18 to $5.51 \mathrm{~g} / \mathrm{d}$, exceeding the reference intake of $2 \cdot 0 \mathrm{~g} \mathrm{Na} / \mathrm{d}^{(4,5)}$.

One of the main determinants of food intake, and potentially salt, is taste ${ }^{(6)}$. The ability to perceive a certain taste may be genetically determined $^{(7)}$. More specifically, genetic variation in taste receptors may alter an individual's taste function ${ }^{(8)}$. However, to our knowledge, only one study reports the genetic predisposition to salt taste in humans. SNP in genes coding for ion channels, the epithelial $\mathrm{Na}$ channel (ENaC, SCNN1B) rs239345 and the transient receptor potential cation subfamily $\mathrm{V}$ member 1 channel (TRPV1) rs8065080, modified the salt taste perception in ninety-five white young adults ${ }^{(8)}$. The effect of these genetic variants on actual $\mathrm{Na}$ intake has not been investigated and the results warrant further investigation. In addition, a link between salt taste perception and blood pressure (BP) is suggested. A number of studies reported that individuals with lower ability to taste salt (i.e. reduced salt taste sensitivity) exhibited higher BP compared with individuals with enhanced ability to perceive salty taste. This was observed both in adults and in children and across different populations ${ }^{(9-12)}$. Moreover, research suggests an association between salt taste sensitivity and salt intake, albeit inconclusive ${ }^{(13,14)}$. Considering the above with the notion that high salt intake is a major risk factor for raised $\mathrm{BP}^{(2,3)}$, it can be hypothesised that reduced salt taste sensitivity would result in higher dietary salt intake and consequently in higher BP.

Furthermore, the mechanisms behind the possible link between salt taste perception and salt intake are unclear and confounded by other metabolic and physiological aspects of salt metabolism. The main confounder is salt sensitivity which is defined as an increase in BP in response to a high dietary salt intake ${ }^{(15)}$. Considering that some individuals do not exhibit such increase, the distinction is made between salt-sensitive and salt-resistant populations ${ }^{(16)}$. Salt sensitivity displays a strong heritable component and the genes involved in $\mathrm{Na}$ transport across the cell membrane have shown a strong effect on salt-

Abbreviations: ABPM, ambulatory blood pressure monitoring device; BP, blood pressure; DBP, diastolic blood pressure; MAP, mean arterial pressure; PP, pulse pressure; SBP, systolic blood pressure; STDT, salt taste detection threshold; STRT, salt taste recognition threshold.

* Corresponding author: L. Pilic, email leta.pilic@stmarys.ac.uk 
sensitive changes in $\mathrm{BP}^{(17,18)}$. Specifically, rs7571842 and rs10177833 in the $S L C 4 A 5$ gene, coding for electrogenic sodium bicarbonate cotransporter 2, have been associated with salt sensitivity in Caucasian hypertensive and normotensive populations ${ }^{(19)}$. In addition to salt taste perception, the TRPV1 gene has been associated with salt sensitivity in animals $^{(20,21)}$. Wang \& Wang ${ }^{(20)}$ have reported that in Dahl salt-sensitive rats on a high-salt diet, TRPV1 expression and function are impaired rendering these rats sensitive to salt load in terms of BP regulation. Furthermore, the TRPV1 rs8065080 is a missense SNP resulting in amino acid change at position 585 , from isoleucine to valine, potentially affecting protein function $^{(22)}$. Cantero-Recasens et al. $^{(23)}$ have tested its functional effect by expressing it in HeLa cells and showed a decreased channel activity in response to two typical TRPV1 stimuli, heat and capsaicin, in TRPV1-Val-585 cells compared with TRPV1Ile-585. The loss of function effect of the rs8065080, together with reduced expression and activity of the TRPV1 reported in salt-sensitive animals, suggests this variant may also be involved in salt sensitivity in humans. Finally, several common variants of the ENaC $S C N N 1 B$ gene, including the rs239345, have been associated with $\mathrm{BP}$ or hypertension in different populations $^{(24,25)}$

Recent research in animals suggests an association between salt taste perception and salt-sensitive hypertension mediated by the renin-angiotensin aldosterone system (RAAS) dysfunction $^{(26)}$. To the best of our knowledge, there are no studies in humans confirming this association. In addition, there are no studies comprehensively exploring the link between salt sensitivity of BP, salt taste perception and intake. Furthermore, salt sensitivity is present in $51 \%$ of hypertensive and $26 \%$ of normotensive populations and it is an independent cardiovascular and mortality risk factor ${ }^{(27,28)}$. Since reduction in salt intake may lead to significant reductions in BP in susceptible individuals ${ }^{(1,2)}$, detecting salt sensitivity in young and healthy individuals may result in more successful prevention of hypertension and consequently $\mathrm{CVD}^{(29)}$

Considering the potential link between salt sensitivity, salt taste perception and dietary salt intake together with the underlying genetic basis, the aim of this study was to explore the genetic predisposition to salt sensitivity, expressed as the BP response to $\mathrm{Na}$ loading, in a healthy adult population and its effects on salt taste perception and dietary salt intake.

\section{Methods}

\section{Subjects}

The subjects were predominantly young Caucasians, eight males and twelve females. Subjects were recruited through advertisements, Internet postings and the institutional centre for workplace and community health. Eligibility criteria were clearly stated. More specifically, subjects were excluded with current stage-2 hypertension (systolic blood pressure (SBP) $\geq 160 \mathrm{mmHg}$ and/or diastolic blood pressure (DBP) $\geq 100 \mathrm{mmHg}$ ), current or recent ( $<1$ month before screening visit) use of antihypertensive medications or medications that affect BP. Further, those with secondary hypertension, history of CVD, chronic kidney failure and current diabetes were excluded. Also excluded were individuals with peptic ulcer disease or liver disease requiring treatment during the previous 2 years. In addition, pregnant women, underweight (BMI $<18.5 \mathrm{~kg} / \mathrm{m}^{2}$ ) and obese (BMI $>30 \mathrm{~kg} / \mathrm{m}^{2}$ ) individuals, individuals exceeding maximal recommended alcohol intake for the UK, those currently adhering to a low-Na diet or with an illness that permanently alters taste were also excluded from the study.

All twenty subjects completed the taste threshold determination test to assess salt taste perception, FFQ and provided a saliva sample. Out of twenty subjects, nineteen completed the low- and high-Na dietary protocols; however, five subjects were excluded due to incomplete 24-h BP or urinary excretion data (Fig. 1).

This study was conducted according to the guidelines laid down in the Declaration of Helsinki, and all procedures involving human subjects were approved by the Institutional Ethics Committee. Written informed consent was obtained from each subject before the baseline data collection informing they can withdraw from the study at any point. The study is registered under Research Registry unique identification number: researchregistry 1652 .

\section{Baseline measurements}

Height and baseline BP and weight were measured during the first examination. Subjects were instructed to avoid alcohol, cigarette smoking, coffee/tea and exercise for at least $30 \mathrm{~min}$ before their BP measurement. Seated BP was measured with an automated BP monitor (Omron M24/7; Meditech Ltd) using an appropriate size cuff after $5 \mathrm{~min}$ of rest. In all, two measurements were performed within 5-min intervals and used for the analysis and calculation of the mean baseline SBP and DBP. In addition, demographic data (age, sex and race) were collected and assessed together with smoking habits and health status information. Physical activity was assessed with the general practice physical activity questionnaire. Participants were considered as active, moderately active, moderately inactive or inactive $^{(30)}$.

\section{Taste thresholds for salt}

Identification of taste thresholds for salt (salt taste perception) was determined using the British Standard BS ISO3972:2011 methodology. Salt taste detection and recognition thresholds were determined using eight graded sodium chloride solutions $(4,6,8,12,17,24,34$ and $49 \mathrm{mmol} / \mathrm{l})$. Solutions were prepared by dissolving food grade sodium chloride in spring water. All solutions were prepared on the day of the testing. Subjects were presented with a sample of each solution by order of increasing concentration starting with the lowest concentration of $4 \mathrm{mmol} / \mathrm{l}$. The procedure was repeated three times. Three additional vessels containing dilutions of the same concentration as the preceding vessel were presented randomly within the sample series. The salt taste detection threshold (STDT) was identified as the lowest concentration of the sample where the subject can 


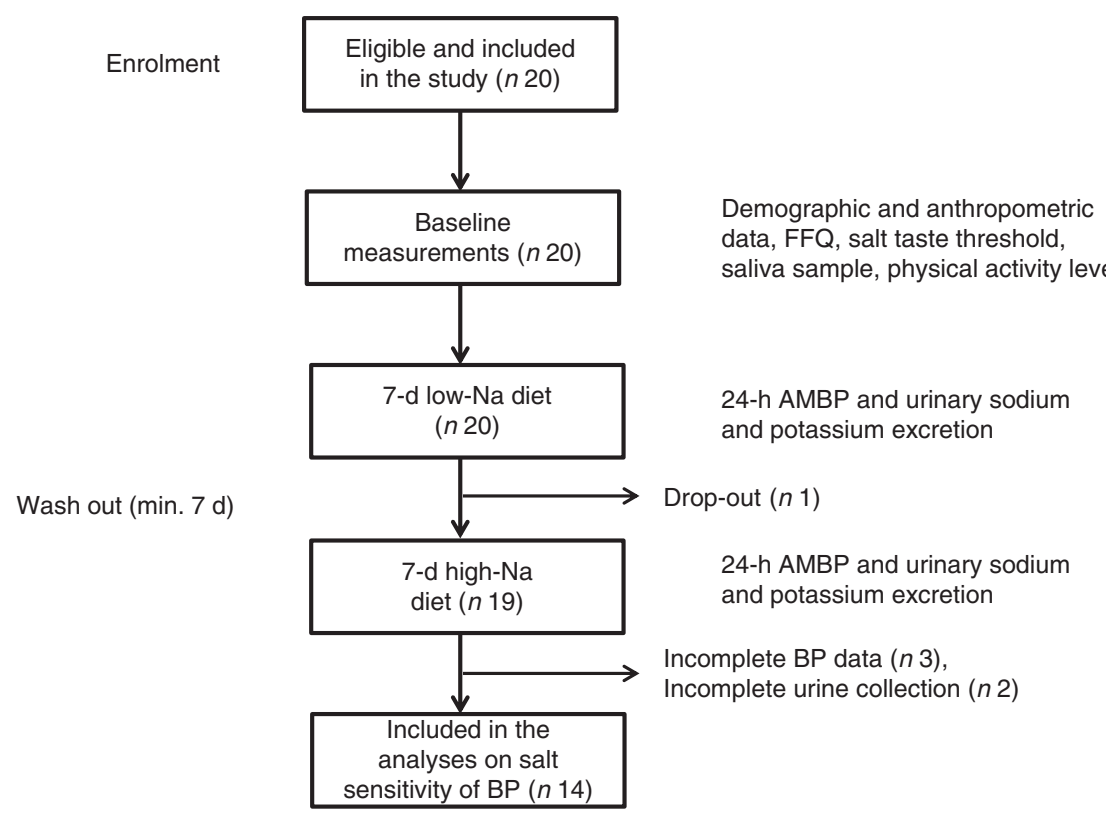

Fig. 1. Overview of the study procedure. ABPM, ambulatory blood pressure monitoring device; BP, blood pressure.

consistently perceive an impression but not identify the taste. The salt taste recognition threshold (STRT) was identified as the sample concentration where the subject consistently perceives the taste as salt ${ }^{(31)}$.

\section{Habitual dietary salt intake}

Baseline energy and dietary salt intake were assessed using a semi-structured validated FFQ. The questionnaires were analysed using the open source, cross-platform tool FETA ${ }^{(32)}$ and information on forty-six nutrients, including $\mathrm{Na}$, was obtained. Habitual dietary $\mathrm{Na}$ intake was energy adjusted and expressed as $\mathrm{mg}$ of $\mathrm{Na} / 4184 \mathrm{~kJ}$ (1000 kcal). Information on the frequency of discretionary salt use was also obtained. Subjects recorded the frequency of adding salt while cooking and at the table by choosing one of the following: (1) never, (2) rarely, (3) sometimes, (4) usually and (5) always.

\section{Dietary sodium intervention}

Study subjects received a low-Na diet ( $3 \mathrm{~g}$ of salt or $51.3 \mathrm{mmol}$ $\mathrm{Na} / \mathrm{d}$ ) for $7 \mathrm{~d}$, followed by a high-Na diet (18g of salt or $307.8 \mathrm{mmol} \mathrm{Na} / \mathrm{d}$ ) for an additional $7 \mathrm{~d}$. Minimal wash-out period between the diets was $7 \mathrm{~d}$. The low-Na diet was designed by investigators using the nutritional analysis software (Nutritics; Nutritics Ltd). A total of three meals and two snacks were designed to provide a total of $3 \mathrm{~g}$ salt/ $\mathrm{d}$ and recommended macronutrient intake ${ }^{(33)}$. Total energy intake was determined based on individual requirements of each subject. Subjects were provided with detailed written instructions about the diets and they were also instructed to maintain their coffee, smoking and physical activity levels. The high-Na diet was formulated by supplementing the low-Na diet with additional $256.5 \mathrm{mmol} \mathrm{Na} / \mathrm{d}$ (15 g salt/d) dispensed by research staff in small paper sachets each containing $1 \mathrm{~g}$ salt $(\mathrm{NaCl})$. To monitor subject compliance with the diets, on the last day of each period, 24-h urine was collected for $\mathrm{Na}, \mathrm{K}$ and creatinine excretion measurements. During the same period, 24-h BP measurements were performed with the 24-h ambulatory blood pressure monitoring device (ABPM).

\section{4-h automated blood pressure monitoring}

In all, 24-h ABPM was attached to the upper, non-dominant arm and $\mathrm{BP}$ was registered at 30-min intervals during daytime and 60-min intervals at night time. Data from the ABPM were downloaded using BP tracker software and mean SBP and DBP were calculated. Subject data with $<30$ successful measurements on each occasion were excluded from the analysis for salt sensitivity ${ }^{(34)}$. Pulse pressure (PP) was calculated according to the formula: $\mathrm{PP}=\mathrm{SBP}-\mathrm{DBP}$ and mean arterial pressure (MAP) as: $\mathrm{MAP}=\mathrm{DBP}+1 / 3 \mathrm{PP}$. Salt sensitivity was defined as an increase of $\geq 3 \mathrm{mmHg}$ in MAP when transitioning from the low- to high-Na diet, as suggested by Kurtz et al. ${ }^{(35)}$. The change in BP between the high-Na and low-Na diet $(\Delta \mathrm{BP})$ was calculated as: $\Delta \mathrm{BP}=$ high-Na diet $\mathrm{BP}-$ low-Na diet BP.

\section{Biochemical measurements}

The 24-h urinary Na and $\mathrm{K}$ were analysed using an automated clinical chemistry analyser (RX Daytona; Randox), with intraassay $\mathrm{CV}<6 \%$. Estimated salt intake was calculated using the equation $17.1 \mathrm{mmol}$ of $\mathrm{Na}=1 \mathrm{~g}$ of salt. Assessment of the completeness of the collection was assessed by measuring creatinine levels from the same urine samples. The following criteria were used: (1) incomplete urine $=<0.7$ of (mmol urinary creatinine $\times 113) /(21 \times \mathrm{kg} \text { of body weight })^{(36)}$ and (2) urinary creatinine $<4 \mathrm{mmol} / \mathrm{d}$ for women, or $<6 \mathrm{mmol} / \mathrm{d}$ for men or a $24 \mathrm{~h}$ urine collection of $<500 \mathrm{ml}$ for either sex and extreme outliers for urinary creatinine (i.e. $>3 \mathrm{sD}$ from the mean) 
considered as unacceptable ${ }^{(37)}$. Subjects with incomplete urine collection from any of the dietary intervention periods, based on any of the two criteria, were excluded from the analysis.

\section{SNP determination}

Following the extensive literature review, four SNP were selected for genotyping: rs7571842 (A/G) and rs10177833 (A/C) in the SLC4A5 gene, rs239345 (T/A) in the SCNN1B and rs8065080 (T/C) in the TRPV1 gene. These SNP were chosen based on their previously reported associations with BP phenotypes, such as hypertension or salt sensitivity, and salt taste perception. This was combined with prevalence data (minor allele frequencies) for the $\mathrm{SNP}^{(8,19,38)}$ (online Supplementary Table S1).

At baseline examination, a 2-ml saliva sample was collected into a collection vial (SalivaGene Collection Module II; Stratec Molecular $\mathrm{GmbH})$. A stabiliser provided by the manufacturer was added to the saliva sample and it was stored at $-20^{\circ} \mathrm{C}$ until DNA was extracted. Genomic DNA was extracted using a commercial kit PSP ${ }^{\circledR}$ SalivaGene 17 DNA Kit 1011 (Stratec Molecular GmbH) in accordance with the manufacturer protocol. Quality and quantity were assessed using Nanodrop (Thermo Fisher Scientific). Genotyping was performed using a predesigned TaqMan ${ }^{\circledR}$ SNP genotyping assays for the SNP: rs7571842, rs10177833, rs239345, rs8065080 and the StepOnePlus thermocycler (Applied Biosystems) with two technical replicates for each sample. The primers and the probes were predesigned by Applied Biosystems with the following codes (C___197439_10, C___1137534_10, C__2387896_30, C_11679656_10). The PCR amplification was performed under the conditions specified by the manufacturer. SNP were accepted when the quality threshold was above $98 \%$. All SNP had minor allele frequencies higher than or equal to $30 \%$ and these reflected the ones reported in European populations ${ }^{(38)}$ (online Supplementary Table S2).

\section{Statistical analysis}

Sample size calculation was based on the $4 \mathrm{mmHg}$ difference in MAP when transitioning from low- to high-Na diet. This difference in BP was observed in other studies investigating salt sensitivity in normotensive populations and with a 24-h $\mathrm{ABPM}^{(39,40)}$. A sample size of fifteen was calculated using an $\alpha$ of 0.05 , power of $80 \%$, expected large effect size $(d=0 \cdot 8)$ and a standard deviation of $5 \mathrm{mmHg}$. This standard deviation was chosen due to lower variability of $\mathrm{BP}$ reported in younger and healthy individuals ${ }^{(40,41)}$.

All continuous variables are presented as means with their standard errors or median (interquartile range). Categorical variables are presented as absolute (relative) frequencies. Before further statistical analysis, continuous variables were tested for normality with the Shapiro-Wilk test. Differences in baseline characteristics by salt sensitivity status were assessed using an independent-samples $t$ test (with Levene's test for equality of variance) or Fisher's exact test. The difference between clinical characteristics of subjects between the lowand high-Na diets was assessed using paired-samples $t$ test. An independent-samples $t$ test (with Levene's test for equality of variance) or Mann-Whitney $U$ test, as appropriate, was used to test for the difference in salt-sensitive changes in BP and dietary $\mathrm{Na}$ intake by genotypes of interest. The model used for the analysis was as follows: major allele homozygote $v$. heterozygote plus minor allele homozygote. A Cochran-Armitage test of trend was run to determine whether a linear trend exists between the genotypes of interest and the proportion of subjects with low and high STDT and STRT as well as the proportion of subjects in different tertiles of energy-adjusted $\mathrm{Na}$ intake. Considering there is no universal cut-off point provided to distinguish between the subjects with low and high salt taste thresholds, a median was used as a cut-off. Subjects with STDT $\leq 8 \mathrm{mmol} / 1$ and STRT $\leq 12 \mathrm{mmol} / \mathrm{l}$ were considered to have low thresholds.

To assess the relationship between salt taste thresholds and salt-sensitive changes in BP and salt taste thresholds and $\mathrm{Na}$ intake, Spearman's correlation analysis was performed. Analyses were performed using the SPSS software package (version 22.0). All tests were two-tailed, with $P<0.05$ considered statistically significant.

\section{Results}

\section{Subject characteristics and compliance with the dietary sodium intervention}

A total of twenty subjects completed the baseline examination, taste threshold determination test and FFQ. Of these, fourteen subjects provided complete 24-h ABPM and 24-h urine excretion data and were included in the analysis on salt sensitivity of BP. A total of five subjects were considered salt-sensitive using the criteria of $\geq 3 \mathrm{mmHg}$ increase in MAP when transitioning from low- to high-Na diet. The study population was normotensive, predominantly white, physically active and nonsmoking with a median age of 28 years (Table 1 ). There was no significant difference in any of the baseline parameters between salt-sensitive and salt-resistant subjects.

In addition, there was no difference in $\mathrm{BP}$ between the low-Na and high-Na diet periods (Table 2). Urinary $\mathrm{Na}$ excretion results demonstrated good compliance with the diet $(P<0.0005)$ whereas $\mathrm{K}$ intake remained similar in both diets $(P=0.243)$.

\section{Genetic predisposition to salt sensitivity of blood pressure, altered salt taste perception and salt intake}

Regarding the genetic predisposition to salt sensitivity, the mean change in BP between the low- and high-Na diet differed according to SLC4A5 rs7571842 genotype (Fig. 2). The subjects with AA genotype had the highest increase in $\mathrm{BP}(\triangle \mathrm{SBP}=7.75$ (sEM 1.44$) \mathrm{mmHg}, \quad P=0.002, \quad d=2 \cdot 4 ; \quad \Delta \mathrm{DBP}=6.25 \quad$ (sEM $2 \cdot 81) \mathrm{mmHg}, P=0.044, d=1.3 ; \Delta \mathrm{MAP}=6.5($ SEM $2 \cdot 10) \mathrm{mmHg}$, $P=0.014, d=1 \cdot 7$ ). SNP rs10177833 (SLC4A5) (Fig. 2), rs239345 (SCNN1B) and rs8065080 (TRPV1) had no statistically significant effects on the BP response to dietary Na manipulation (data not shown). Moreover, the analysis was conducted to test for the possible difference in the prevalence of males and females, BMI and age, between the rs7571842 genotype groups. There was no difference in any of the variables between the AA and $\mathrm{AG}+\mathrm{GG}$ group $(P=1.000, P=0.846$ and $P=0.584$ for sex, BMI and age, respectively). 
Table 1. Baseline characteristics of study subjects, total sample ( $n 20)$ and according to salt sensitivity status ( $n$ 14) ${ }^{\star}$ (Mean values with their standard errors; absolute and relative frequencies; medians and interquartile ranges)

\begin{tabular}{|c|c|c|c|c|c|c|c|}
\hline & \multicolumn{2}{|c|}{ Total $(n 20)$} & \multicolumn{2}{|c|}{ Salt-sensitive $(n 5)$} & \multicolumn{2}{|c|}{ Salt-resistant $(n 9)$} & \multirow[b]{2}{*}{$P$} \\
\hline & Absolute frequency & Relative frequency & Absolute frequency & Relative frequency & Absolute frequency & Relative frequency & \\
\hline Age (years) & & & & & & & 0.612 \\
\hline Median & 28.0 & & $35 \cdot 8$ & & 33.2 & & \\
\hline Interquartile range & $10 \cdot 5$ & & 4.6 & & $2 \cdot 7$ & & \\
\hline \multicolumn{8}{|l|}{ Sex } \\
\hline Male & 8 & 40 & 2 & 40 & 2 & 22 & 0.580 \\
\hline Female & 12 & 60 & 3 & 60 & 7 & 78 & \\
\hline \multicolumn{8}{|l|}{ Race } \\
\hline White & 16 & 80 & 4 & 80 & 6 & 67 & 0.999 \\
\hline Other & 4 & 20 & 1 & 20 & 3 & 33 & \\
\hline BMI $\left(\mathrm{kg} / \mathrm{m}^{2}\right)$ & & & & & & & 0.633 \\
\hline Mean & 23.9 & & $24 \cdot 7$ & & $23 \cdot 7$ & & \\
\hline SEM & 0.7 & & 1.9 & & 0.7 & & \\
\hline SBP (mmHg) & & & & & & & 0.413 \\
\hline Mean & $121 \cdot 3$ & & $125 \cdot 8$ & & $118 \cdot 2$ & & \\
\hline SEM & $3 \cdot 0$ & & $9 \cdot 2$ & & 4.4 & & \\
\hline DBP $(\mathrm{mmHg})$ & & & & & & & 0.913 \\
\hline Mean & $70 \cdot 4$ & & 71.9 & & $71 \cdot 2$ & & \\
\hline SEM & $2 \cdot 1$ & & $6 \cdot 3$ & & 2.9 & & \\
\hline \multicolumn{8}{|l|}{ Smoking status } \\
\hline Yes & 1 & 5 & 1 & 20 & 0 & 0 & 0.357 \\
\hline No & 19 & 95 & 4 & 80 & 9 & 100 & \\
\hline \multicolumn{8}{|l|}{ Physical activity level } \\
\hline Active & 15 & 75 & 2 & 40 & 7 & 78 & 0.413 \\
\hline Moderately active & 1 & 5 & 1 & 20 & 0 & & \\
\hline Moderately inactive & 2 & 10 & 1 & 20 & 1 & 11 & \\
\hline Inactive & 2 & 10 & 1 & 20 & 1 & 11 & \\
\hline
\end{tabular}

DBP, diastolic blood pressure; SBP, systolic blood pressure.

${ }^{\star} P$ value for difference between salt-sensitive and salt-resistant subjects (independent-samples $t$ test, Fisher's exact test).

Table 2. Clinical characteristics of study subjects $(n 14)$ on low- and high-salt diet ${ }^{\star}$ (Mean values with their standard errors)

\begin{tabular}{|c|c|c|c|c|c|}
\hline & \multicolumn{2}{|c|}{ Low-salt diet } & \multicolumn{2}{|c|}{ High-salt diet } & \multirow[b]{2}{*}{$P$} \\
\hline & Mean & SEM & Mean & SEM & \\
\hline $\mathrm{SBP}(\mathrm{mmHg})$ & $113 \cdot 6$ & $2 \cdot 7$ & $115 \cdot 8$ & $3 \cdot 0$ & 0.107 \\
\hline $\mathrm{DBP}(\mathrm{mmHg})$ & 66.9 & 1.4 & $68 \cdot 6$ & $2 \cdot 2$ & 0.261 \\
\hline MAP (mmHg) & $82 \cdot 5$ & 1.6 & 84.4 & $2 \cdot 4$ & 0.170 \\
\hline $\mathrm{PP}(\mathrm{mmHg})$ & $46 \cdot 7$ & $2 \cdot 2$ & $47 \cdot 2$ & 1.8 & 0.656 \\
\hline Urine $\mathrm{Na}$ excretion (mmol/24 h) & $66 \cdot 1$ & 8.9 & 281.5 & 24.4 & $3.3 \times 10^{-1}$ \\
\hline Urine $\mathrm{K}$ excretion (mmol/24 h) & 75.8 & $5 \cdot 5$ & $81 \cdot 8$ & $5 \cdot 8$ & 0.243 \\
\hline
\end{tabular}

SBP, systolic blood pressure; DBP, diastolic blood pressure; MAP, mean arterial pressure, PP, pulse pressure.

${ }^{*} P$ values for difference between low- and high-salt diets (paired-samples $t$ test).

In contrast with the above described, the proportion of study subjects with low and high STRT was similar according to genotypes of interest (Fig. 3). The results of a Cochran-Armitage test of trend between the different genotype groups (homozygous major allele, heterozygous and homozygous minor allele) and the proportion of subjects with low and high STRT were: $r$ rs7571842 $(P=0.905)$, rs10177833 $(P=0.714)$, rs239345 $(P=0.456)$, rs8065080 $(P=0.078)$. Similar were observed for STDT (data not shown). However, a linear trend was observed regarding the distribution of subjects in the first or second + third tertiles of energy-adjusted $\mathrm{Na}$ intake according to the SLC4A5 rs10177833. With the increasing number of A alleles, $\mathrm{Na}$ intake increased $(P=0 \cdot 037$, Fig. 4). The mean age and BMI as well as the distribution of sex did not differ between the rs10177833 genotype groups $(P=0 \cdot 129, P=0 \cdot 551, P=1 \cdot 000$ for age, BMI and sex, respectively).

\section{Associations between salt sensitivity of blood pressure, salt taste perception and salt intake}

When exploring the associations between the main outcome variables, there was no correlation between the mean change in SBP, DBP and MAP, when transitioning from a low- to high-Na diet, and salt taste thresholds (Table 3). However, a positive moderate correlation was observed between the mean change in PP and STDT $\left(r_{\mathrm{S}} 0.551, P=0 \cdot 041\right)$. Sub-group analysis revealed a strong positive correlation between the change in PP and STDT in 
(a)

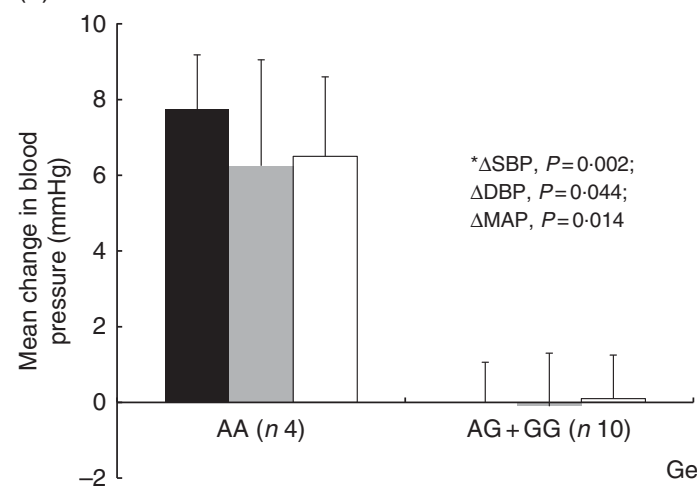

(b)

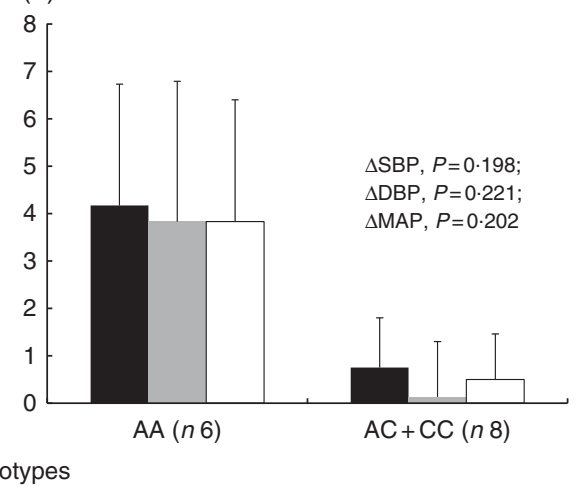

Fig. 2. Systolic blood pressure (SBP), diastolic blood pressure (DBP) and mean arterial pressure (MAP) change from low- to high-salt diet according to SLC4A5 rs7571842 (a) and rs10177833 (b) genotype status ( $n$ 14). Analysis conducted on the following model: major allele homozygote $v$. heterozygote plus minor allele homozygote. Values are means, with their standard errors represented by vertical bars (independent-samples $t$ test, ${ }^{\star}$ Mann-Whitney $U$ test). $\square$, Systolic blood pressure; $\square$, diastolic blood pressure; $\square$, mean arterial pressure.

(a)

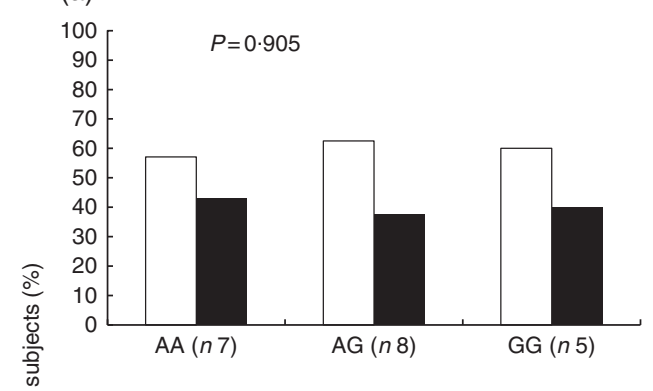

(c)

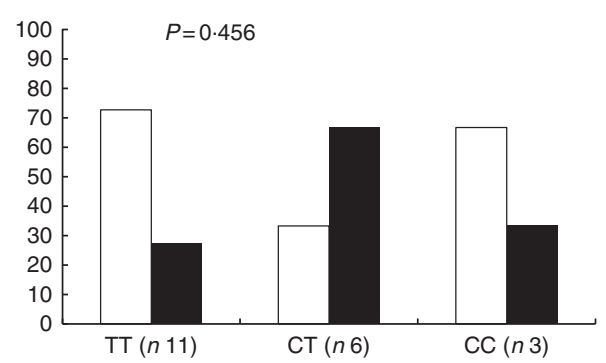

(b)

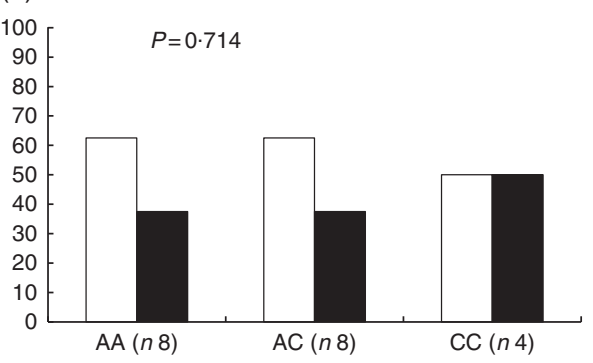

(d)

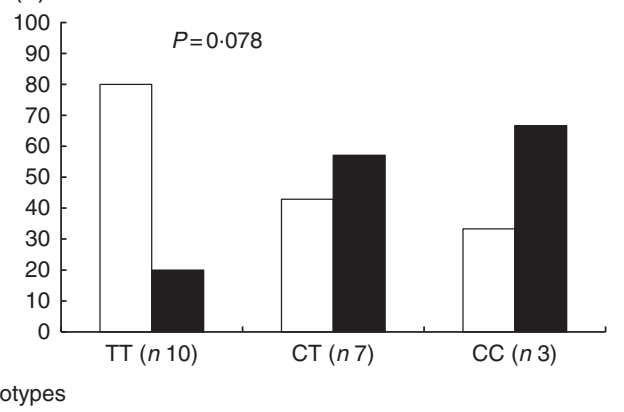

Fig. 3. Proportion of subjects ( $n$ 20) with low and high salt taste recognition thresholds according to SLC4A5 rs7571842 (a) and rs10177833 (b), SCNN1B rs239345 (c) and TRPV1 rs8065080 (d) genotype. Low threshold $(\square)$ and high threshold $(\square)$ (Cochran-Armitage test of trend).

the SLC4A5 rs7571842 AG + GG group $\left(r_{\mathrm{s}} 0 \cdot 845, P=0 \cdot 002\right)$. Similar was observed for the rs10177833. There was a strong positive correlation between the change in PP and STDT in the AC+CC group $\left(r_{\mathrm{s}} 0 \cdot 781, P=0 \cdot 022\right.$, online Supplementary Table S3).

Furthermore, in the total study population, the correlation between the STDT and energy-adjusted $\mathrm{Na}$ intake was not significant $\left(r_{\mathrm{s}} 0.069, P=0.774\right)$. Similar was observed for STRT ( $\left.r_{\mathrm{s}} 0.025, P=0.918\right)$. In addition, the correlation between adding salt while cooking and at the table and salt taste thresholds was also investigated. No significant correlation was observed (STDT: $r_{\mathrm{s}} 0.134, P=0.573$ for adding salt at the table and $r_{\mathrm{s}} 0.342, P=0.140$ for adding salt while cooking; STRT: $r_{\mathrm{s}} 0.083$, $P=0.727$ for adding salt at the table and $r_{\mathrm{s}}-0.071, P=0.767$ for adding salt while cooking, online Supplementary Table S4). However, as shown in Fig. 5, when stratifying according to genotype, in the AA group of the SLC4A5 rs7571842, a strong and positive correlation was observed between adding salt while cooking and both STDT $\left(r_{\mathrm{s}} 0 \cdot 868, P=0 \cdot 011\right)$ and STRT $\left(r_{\mathrm{s}} 0.868, P=0 \cdot 011\right)$. In addition, in the TT group of the TRPV1 rs8065080, a moderate and negative correlation was observed between adding salt at the table and STRT $\left(r_{\mathrm{s}}-0 \cdot 636, P=0.048\right)$.

\section{Discussion}

Genetics of the blood pressure response to sodium loading, salt taste perception and salt intake

Findings from the present study suggest a genetic predisposition to salt sensitivity in the study population. Despite the small sample size, salt-sensitive increase in BP was detected. Moreover, 

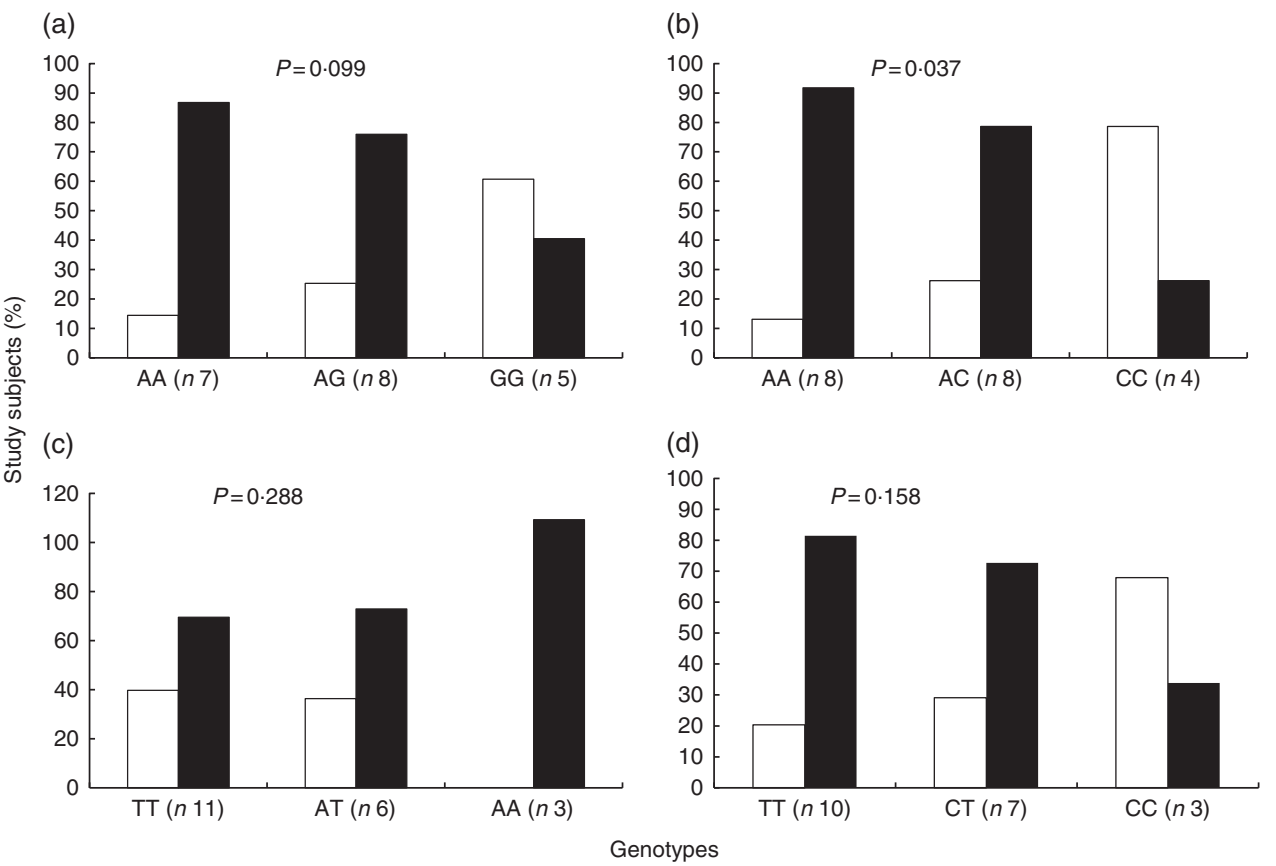

Fig. 4. Proportion of subjects ( $n 20)$ in the different tertiles of energy-adjusted sodium intake according to SLC4A5 rs7571842 (a) and rs10177833 (b), SCNN1B rs239345 (c) and TRPV1 rs8065080 (d) genotype. First tertile $(\square,<1241 \mathrm{mg} / 4184 \mathrm{~kJ}(1000 \mathrm{kcal}))$ and second + third tertiles combined $(\square, \geq 1241 \mathrm{mg} / 4184 \mathrm{~kJ}(1000 \mathrm{kcal}))$ (Cochran-Armitage test of trend).

Table 3. Correlation analysis between salt taste thresholds ( $\mathrm{mol} / \mathrm{l})$ and mean change in BP ( $\mathrm{mmHg}$ ) from low-to high-salt diet, and salt taste thresholds (mol/l) and dietary sodium intake $(\mathrm{mg}$ sodium $/ 4184 \mathrm{~kJ}(1000 \mathrm{kcal}))(n 14)^{\star}$

\begin{tabular}{|c|c|c|c|c|c|c|c|c|c|c|}
\hline & \multicolumn{2}{|c|}{$\Delta \mathrm{SBP}$} & \multicolumn{2}{|c|}{$\triangle \mathrm{DBP}$} & \multicolumn{2}{|c|}{$\triangle \mathrm{MAP}$} & \multicolumn{2}{|c|}{$\Delta \mathrm{PP}$} & \multicolumn{2}{|c|}{$\mathrm{Na}$ intake } \\
\hline STDT & 0.098 & 0.740 & -0.377 & 0.185 & -0.303 & 0.293 & 0.551 & 0.041 & -0.016 & 0.956 \\
\hline STRT & 0.403 & 0.153 & 0.209 & 0.473 & 0.260 & 0.370 & 0.039 & 0.895 & -0.113 & 0.700 \\
\hline
\end{tabular}

SBP, systolic blood pressure; DBP, diastolic blood pressure; MAP, mean arterial pressure; PP, pulse pressure; STDT, salt taste detection threshold; STRT, salt taste recognition threshold.

* Spearman's $\rho$ ( $P$ value)

other studies with similar sample sizes, fourteen to sixteen subjects, respectively, have successfully investigated and detected this phenomenon in normotensive populations ${ }^{(42-44)}$. Finally, urinary markers of compliance with the diets, $\mathrm{Na}$ and $\mathrm{K}$, were satisfactory showing an overall good compliance with the diets.

SLC4A5 gene, coding for a sodium hydrogen bicarbonate transporter involved in $\mathrm{Na}$ transport across the cellular membrane ${ }^{(45)}$, affected salt-sensitive changes in BP. Carey et al. ${ }^{(19)}$ noted that SNP rs7571842 and rs10177833 had the most pronounced effects on salt sensitivity. One of these SNP, rs7571842, had the greatest effect in this study population, increasing BP in individuals with AA genotype and confirming the protective effect of the $G$ allele ${ }^{(19)}$. A post hoc power calculation revealed that, with the two-tailed 0.05 significance level, this test had a power of $92 \%$ to detect a difference in SBP between the two SLC4A5 rs7571842 genotype groups (mean values for $\Delta \mathrm{SBP}$ $7.75 v .0 .00 \mathrm{mmHg}$ and standard deviations $2.87 v .1 .06 \mathrm{mmHg}$ ). Regarding the rs10177833, the lack of confirmation of its effect may be due to its lower effect size that could potentially be detected in a larger sample size study. These results, however, align with Carey et al. ${ }^{(19)}$ where the effect of rs10177833 on salt sensitivity observed in the University of Virginia discovery cohort was not replicated in an International Hypertensive Pathotype (HyperPATH) study population. Other SNP investigated in the present study were not associated with salt sensitivity in previous studies conducted in humans. The SCNN1B SNP were associated with hypertension ${ }^{(24,25)}$ but not salt sensitivity per se suggesting rs239345 may not have an effect on this specific phenotype in a healthy population. Finally, the TRPV1 rs8065080 appears to be functional and is associated with lower channel activity, a trait observed in salt-sensitive rats ${ }^{(20,23)}$. In this population, it did not have an effect on salt-sensitive changes in BP, suggesting that other variants in this gene may have more pronounced effects on BP.

Nevertheless, the A allele of the SLC4A5 rs7571842 is present in approximately half of the European descent population with a third of the population having the risky AA genotype ${ }^{(38)}$. Additionally, salt-sensitive rise in BP, following a high-Na diet, was expressed as a continuous variable. The risk of CVD increases continuously and with each $2 \mathrm{mmHg}$ increase in SBP there is a $7 \%$ increase in risk of mortality from IHD and a $10 \%$ increase in the risk of mortality from stroke ${ }^{(46)}$. The increase in SBP in healthy subjects with the rs7571842 AA genotype was $7.75 \mathrm{mmHg}$, which emphasises the clinical relevance of these 

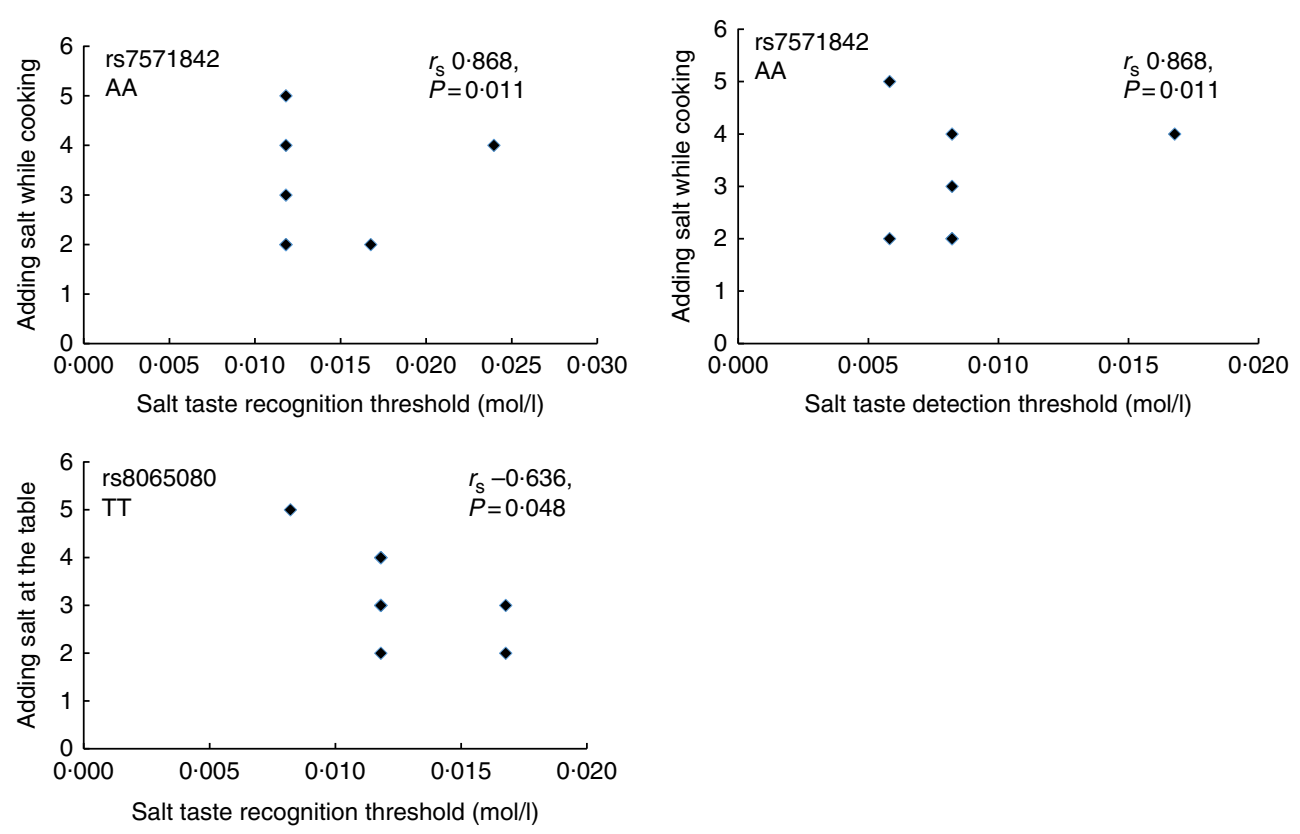

Fig. 5. Correlation between salt taste thresholds and discretionary salt use according to SLC4A5 rs7571842 $(n)$ 6) and TRPV1 rs8065080 ( $n$ 10) genotypes. Adding salt while cooking/table; 1 - always, 2 - usually, 3 - sometimes, 4 - rarely, 5 - never (Spearman's correlation).

results. Moreover, it has been estimated that approximately a third of deaths attributed to BP occur in individuals with BP lower than the hypertensive range ${ }^{(47)}$. They may represent a salt-sensitive part of the population which reflects salt sensitivity prevalence of $36 \%$ in this study. Considering the discrepancies in methods used in previous studies, it is difficult to draw any conclusion whether this prevalence could be expected in other populations with similar characteristics. Salt sensitivity prevalence of $26 \%$ in normotensives was established using an intravenous protocol for diagnosis of salt sensitivity ${ }^{(27)}$. However, more recent work suggests that this method can lead to misclassification and incorrect diagnosis ${ }^{(39,40)}$. Another potential issue in comparison of different study results is the $\mathrm{BP}$ measurement. While most studies still use the conventional measurements, from the studies that employ 24-h BP measurements only a limited number is investigating salt sensitivity solely in healthy, normotensive populations ${ }^{(48-50)}$.

It should be noted, however, that this study primarily investigated the effects of Na loading on BP and as such, the abovedescribed salt sensitivity prevalence should be regarded with caution. When identifying subjects as salt-sensitive or saltresistant it is recommended that the low- and high-Na diets should be administered in a random order to achieve maximal reproducibility ${ }^{(35)}$. When a low $\mathrm{Na}$ period precedes high $\mathrm{Na}$ period RAAS may not be uniformly suppressed ${ }^{(51)}$. This may result in an increased BP response on a low-Na diet and would require larger sample size compared with the one in this study to detect the true effect of dietary Na manipulation on BP and estimate the salt sensitivity prevalence. Therefore, if the order of the diets was randomised and high-Na diet preceded the low$\mathrm{Na}$ diet in a proportion of the study population, the RAAS may have been supressed to an extent where more uniformity in the $\mathrm{BP}$ response to dietary intervention may have been observed. This in turn, may have resulted in a statistically significant difference in BP when transitioning from the low- to the high$\mathrm{Na}$ diet in the total study population.

Besides observed genetic predisposition to salt sensitivity of BP, the SLC4A5 rs10177833 was associated with salt intake. With increasing number of A alleles, there was a trend towards an increased energy-adjusted $\mathrm{Na}$ intake. The highest proportion of subjects in the second and third tertiles of energy-adjusted $\mathrm{Na}$ intake was in the AA genotype group with the majority of these subjects (85\%) having absolute Na intake above the recommendations ${ }^{(5,52)}$. Recently, Smith et al. ${ }^{(53)}$ have reported how individuals with enhanced bitter taste perception genotype (GC and GG alleles for the bitter taste receptor gene TAS2R38) were significantly more likely than CC homozygotes to have daily $\mathrm{Na}$ intake higher than recommended. Furthermore, Kho et al. ${ }^{(54)}$, in their genomewide association study, have reported on several variants associated with salt intake. These variants were in genes coding for $\mathrm{Na}$, $\mathrm{K}$ and $\mathrm{Ca}$ channels, suggesting that genes coding for $\mathrm{Na}$ transport proteins may be associated with increased salt intake, similar to the findings of this study. The mechanism behind this association is to be explored. It is not to exclude the potential expression of this cotransporter in taste receptor cells, as other Na-dependent transporters primarily expressed in other tissues have been localised in tongue ${ }^{(55,56)}$. However, impaired Na metabolism was reported as a consequence of rs10177833 induced increase in the SLC4A5 transcription under conditions of high $\mathrm{Na}$ intake ${ }^{(57)}$. Considering its strong linkage disequilibrium with $\mathrm{rs} 7571842^{(19)}$, these two SNP are most likely inherited together making the carriers of this genotype at increased risk of developing hypertension and CVD.

Moreover, there was no genetic predisposition to altered salt taste perception. The discrepancy in the results of the present study and the one by Dias et $a l^{(8)}$ may be explained by the difference in thresholds measured. The taste quality of salt stimulus can be concentration dependent ${ }^{(58,59)}$ which may explain the associations observed with suprathresholds in Dias 
et al. ${ }^{(8)}$ but not with lower concentrations (STDT, STRT) used in this study. Nevertheless, the borderline non-significant trend observed for the TRPV1 rs8065080 may be detected in a larger sample size study. For such study to be clinically meaningful, in addition to salt taste perception, dietary salt intake should be measured, as acknowledged by Dias et $a l^{(8)}$. It has been shown that the reduction in salt intake results in important falls in BP, in both hypertensive and normotensive salt-sensitive individuals ${ }^{(2)}$, and a reduction in overall CVD risk $^{(1)}$.

\section{Associations between salt sensitivity, salt taste perception and salt intake}

Together with the observed effect of genetics, salt sensitivity expressed as a change in BP after Na loading was associated with taste thresholds for salt. In subjects that had complete dietary intervention data, PP was positively associated with STDT. PP is the difference between SBP and DBP and is argued to be a better predictor of cardiovascular risk than $\mathrm{SBP}^{(60)}$. $\mathrm{PP}$ may be genetically determined by the SLC4A5 rs7571842(61). The mechanisms behind this association and the causality remain unknown. However, the hypothesis was that genetics may play a role in this relationship which aligns with the finding that this association was observed only in certain genotype groups of the SLC4A5 SNP. This subgroup analysis should, nevertheless, be replicated in a study with a larger sample size in each genotype group, to achieve appropriate statistical power, and as such considered preliminary in this study.

Sakamoto et $a l .{ }^{(26)}$ reported that the $\mathrm{ENaC}$ activity may be the link between salt taste sensitivity and salt sensitivity of BP in animals. However, the $S C N N 1 B$ rs239345 was not associated with salt sensitivity or salt taste thresholds in this study. In a larger sample size study potential effect of interactions between the SLC4A5 and ENaC SNP may be investigated and may provide insight into the mechanism behind this relationship. Nevertheless, the relevance of these findings lies in the actual relationship between salt taste thresholds and salt intake.

If there is a positive association between the thresholds for salt and salt-sensitive changes in $\mathrm{BP}$, it can be theorised that saltsensitive individuals with higher thresholds are at greater risk of developing hypertension due to their higher salt intake. In the present study, however, neither detection nor the recognition threshold for salt has been associated with total habitual dietary salt intake. Nevertheless, discretionary salt use accounts for approximately $15 \%$ of salt intake in Western countries ${ }^{(62)}$ and the results of the present study suggest it may be associated with salt taste thresholds. The association between salt taste perception and discretionary salt use may depend on the SLC4A5 and TRPV1 genotype, however these sub-group analyses should be replicated in a larger size study. This would, nonetheless, be in line with the notion that reduced salt taste sensitivity (i.e. higher salt taste threshold) drives individuals to consume more salt until reaching the salt concentration identified as pleasant ${ }^{(14)}$. Conversely, improved ability to taste salt when the taste of salt is deemed pleasant may result in increased salt intake. Indeed, research suggests that the preference for salty taste may be one of the factors affecting salt intake in younger populations and that discretionary salt use is more frequent in younger compared with older populations ${ }^{(63,64)}$. Moreover, when salt content of processed food is reduced, consumers compensate its apparent lack by increasing the discretionary salt use ${ }^{(65)}$. Considering the evolving food supply and dietary habits of the UK population and worldwide, a better understanding of this behaviour could enable more targeted and effective public health interventions to reduce salt intake.

\section{Strengths and limitations}

This study has several strengths and limitations. A strength is the salt sensitivity phenotyping procedure with the dietary control of $\mathrm{Na}$ intake. Moreover, a 24-h ABPM procedure to determine the difference in BP between the diets provides many more measurements than conventional BP measurement reflecting usual BP more accurately. It also allows identification of individuals with a 'white coat' response or masked hypertension, and is a stronger predictor of cardiovascular morbidity and mortality than conventional measurement ${ }^{(34)}$. One of the limitations is a use of a FFQ to determine dietary salt intake. Even though FFQ represents dietary intake over a longer time-period, it relies heavily on respondents' honesty and long-term memory. However, Na intake was energy adjusted, improving measurement accuracy. Freedman et al. ${ }^{(6)}$ suggest that the attenuations and correlations with truth for the FFQ are improved when considering $\mathrm{Na}$ densities, utilised in this study. Regarding the associations between genetics and variables of interest, where possible, a CochranArmitage test of trend was used. The advantage of the CochranArmitage trend test is that it is not dependent on the HardyWeinberg equilibrium assumption and is suggested as the genotype-based test for association ${ }^{(67-69)}$. Finally, the small sample size in subgroup analyses of the correlations between salt taste perception, $\mathrm{BP}$ response to $\mathrm{Na}$ loading and salt intake warrants replication of these results in a larger sample size study.

In conclusion, this preliminary data suggest there is a genetic predisposition to salt sensitivity in healthy, adult Caucasians. The SLC4A5 rs7571842 was confirmed as the variant with the effect on salt-sensitive changes in BP. Another SLC4A5 variant, rs10177833, most likely inherited together with the rs7571842, is associated with salt intake. Moreover, the observed associations between salt taste perception and salt sensitivity, together with the association between salt taste perception and discretionary salt use may depend on the SLC4A5 and TRPV1 genotype. As there was no association between genetics and salt taste perception, the mechanisms behind these associations are to be further explored together with gene-gene interactions. Nevertheless, preference for salty taste may be a driver of salt intake in younger populations and warrants further investigation. Studies investigating these associations should comprehensively explore all potential variables, such as genetic predisposition, salt taste perception and salt intake to contribute towards more successful prevention of hypertension and CVD.

\section{Acknowledgements}

The authors would like to thank Professor Conor Gissane for his advice on the final version of this manuscript. The authors would like to acknowledge the contribution of the staff and 
subjects of the EPIC-Norfolk Study. EPIC-Norfolk is supported by the Medical Research Council programme grants (G0401527, G1000143) and Cancer Research UK programme grant (C864/ A8257).

This research received no specific grant from any funding agency, commercial or not-for-profit sectors.

Y. M. and L. P. designed the experiment. L. P. conducted data collection, data analysis and wrote the manuscript. Y. M. supervised the project. Both authors discussed the results and implications and commented on the manuscript at all stages.

The authors declare that there are no conflicts of interest.

\section{Supplementary material}

For supplementary material/s referred to in this article, please visit https://doi.org/10.1017/S0007114518002027

\section{References}

1. Lewington S, Clarke R, Qizilbash N, et al. (2002) Age-specific relevance of usual blood pressure to vascular mortality: a meta-analysis of individual data for one million adults in 61 prospective studies. Lancet 360, 1903-1913.

2. He FJ, Li J \& MacGregor GA (2013) Effect of longer term modest salt reduction on blood pressure: Cochrane systematic review and meta-analysis of randomised trials. BMJ $\mathbf{3 4 6}$, f1325.

3. He FJ \& MacGregor GA (2004) Effect of longer-term modest salt reduction on blood pressure. Cochrane Database Syst Rev, issue 3, CD004937.

4. Mozaffarian D, Fahimi S, Singh GM, et al. (2014) Global sodium consumption and death from cardiovascular causes. $N$ Engl J Med 371, 624-634.

5. World Health Organization (2012) Guideline: Sodium Intake for Adults and Children. Geneva: WHO.

6. Sørensen LB, Møller P, Flint A, et al. (2003) Effect of sensory perception of foods on appetite and food intake: a review of studies on humans. Int J Obes 27, 1152-1166.

7. Reed DR, Tanaka T \& McDaniel AH (2006) Diverse tastes: genetics of sweet and bitter perception. Physiol Behav 88, 215-226.

8. Dias AG, Rousseau D, Duizer L, et al. (2013) Genetic variation in putative salt taste receptors and salt taste perception in humans. Chem Senses 38, 137-145.

9. Okoro EO, Uroghide GE \& Jolayemi ET (1998) Salt taste sensitivity and blood pressure in adolescent school children in southern Nigeria. East Afr Med J 75, 199-203.

10. Isezuo SA, Saidu Y, Anas S, et al. (2008) Salt taste perception and relationship with blood pressure in type 2 diabetics. J Hum Hypertens 22, 432-434.

11. Rabin M, Poli de Figueiredo CE, Wagner MB, et al. (2009) Salt taste sensitivity threshold and exercise-induced hypertension. Appetite 52, 609-613.

12. Kirsten VR \& Wagner MB (2014) Salt taste sensitivity thresholds in adolescents: are there any relationships with body composition and blood pressure levels? Appetite 81, 89-92.

13. Azinge EC, Sofola OA \& Silva BO (2011) Relationship between salt intake, salt-taste threshold and blood pressure in Nigerians. West Afr J Med 30, 373-376.

14. Piovesana PM, Sampaio KL \& Gallani MCBJ (2013) Association between taste sensitivity and self-reported and objective measures of salt intake among hypertensive and normotensive individuals. ISRN Nutr 2013, 301213.
15. Sullivan JM (1991) Salt sensitivity. Definition, conception, methodology, and long-term issues. Hypertension 17, I61-I68.

16. Weinberger MH (1996) Salt sensitivity of blood pressure in humans. Hypertension 27, 481-490.

17. Hunt SC, Xin Y, Wu LL, et al. (2006) Sodium bicarbonate cotransporter polymorphisms are associated with baseline and 10-year follow-up blood pressures. Hypertension $\mathbf{4 7}$, 532-536.

18. Yang X, He J, Gu D, et al. (2014) Associations of epithelial sodium channel genes with blood pressure changes and hypertension incidence: the GenSalt study. Am J Hypertens 27, 1370-1376.

19. Carey RM, Schoeffel CD, Gildea JJ, et al. (2012) Salt sensitivity of blood pressure is associated with polymorphisms in the sodium-bicarbonate cotransporter. Hypertension $\mathbf{6 0}$, 1359-1366.

20. Wang Y \& Wang DH (2006) A novel mechanism contributing to development of Dahl salt-sensitive hypertension: role of the transient receptor potential vanilloid type 1. Hypertension $\mathbf{4 7}$, 609-614.

21. Hao X, Chen J, Luo Z, et al. (2011) TRPV1 activation prevents high-salt diet-induced nocturnal hypertension in mice. Pflüg Arch Eur J Physiol 461, 345-353.

22. Ng PC \& Henikoff S (2006) Predicting the effects of amino acid substitutions on protein function. Annu Rev Genomics Hum Genet 7, 61-80.

23. Cantero-Recasens G, Gonzalez JR, Fandos C, et al. (2010) Loss of function of transient receptor potential vanilloid 1 (TRPV1) genetic variant is associated with lower risk of active childhood asthma. J Biol Chem 285, 27532-27535.

24. Hannila-Handelberg T, Kontula K, Tikkanen I, et al. (2005) Common variants of the beta and gamma subunits of the epithelial sodium channel and their relation to plasma renin and aldosterone levels in essential hypertension. BMC Med Genet 6, 4

25. Jin H-S, Hong K-W, Lim J-E, et al. (2010) Genetic variations in the sodium balance-regulating genes ENaC, NEDD4L, NDFIP2 and USP2 influence blood pressure and hypertension. Kidney Blood Press Res 33, 15-23.

26. Sakamoto T, Fujii A, Saito N, et al. (2016) Alteration of amiloride-sensitive salt taste nerve responses in aldosterone/ $\mathrm{NaCl}$-induced hypertensive rats. Neurosci Res 108, 60-66.

27. Weinberger MH, Miller JZ, Luft FC, et al. (1986) Definitions and characteristics of sodium sensitivity and blood pressure resistance. Hypertension 8, 127-134.

28. Weinberger MH, Fineberg NS, Fineberg SE, et al. (2001) Salt sensitivity, pulse pressure, and death in normal and hypertensive humans. Hypertension 37, 429-432.

29. Iatrino R, Manunta P \& Zagato L (2016) Salt sensitivity: challenging and controversial phenotype of primary hypertension. Curr Hypertens Rep 18, 70.

30. Department of Health (2013) General practice physical activity questionnaire (GPPAQ). https://www.gov.uk/government/ publications/general-practice-physical-activity-questionnairegppaq (accessed April 2018).

31. British Standards Institution (2011) Sensory Analysis. Methodology. Method of Investigating Sensitivity of Taste. Geneva: ISO (BS ISO 3972:2011).

32. Mulligan AA, Luben RN, Bhaniani A, et al. (2014) A new tool for converting food frequency questionnaire data into nutrient and food group values: FETA research methods and availability. BMJ Open 4, e004503.

33. Department of Health (1991) Dietary Reference Values for Food Energy and Nutrients for the United Kingdom: Report of the Panel on Dietary Reference Values of the Committee on Medical Aspects of Food Policy. London: The Stationery Office. 
34. O'Brien E, Parati G \& Stergiou G (2013) Ambulatory blood pressure measurement what is the international consensus? Hypertension 62, 988-994.

35. Kurtz TW, DiCarlo SE, Pravenec M, et al. (2017) An appraisal of methods recently recommended for testing salt sensitivity of blood pressure. J Am Heart Assoc 6, e005653.

36. Murakami K, Sasaki S, Takahashi Y, et al. (2008) Sensitivity and specificity of published strategies using urinary creatinine to identify incomplete 24-h urine collection. Nutrition 24, 16-22.

37. Land MA, Webster J, Christoforou A, et al. (2014) Salt intake assessed by $24 \mathrm{~h}$ urinary sodium excretion in a random and opportunistic sample in Australia. BMJ Open 4, e003720.

38. National Institutes of Health (2017) dbSNP - short genetic variations. https://www.ncbi.nlm.nih.gov/projects/SNP/ (accessed January 2017).

39. de la Sierra A, Giner V, Bragulat E, et al. (2002) Lack of correlation between two methods for the assessment of salt sensitivity in essential hypertension. J Hum Hypertens 16, 255-260.

40. Sharma AM, Schorr U, Cetto C, et al. (1994) Dietary $v$. intravenous salt loading for the assessment of salt sensitivity in normotensive men. Am J Hypertens 7, 1070-1075.

41. Mancia G (2012) Short- and long-term blood pressure variability. Hypertension 60, 512-517.

42. Scuteri A, Stuehlinger MC, Cooke JP, et al. (2003) Nitric oxide inhibition as a mechanism for blood pressure increase during salt loading in normotensive postmenopausal women. J Hypertens 21, 1339-1346.

43. Luft FC, Rankin LI, Bloch R, et al. (1979) Cardiovascular and humoral responses to extremes of sodium intake in normal black and white men. Circulation 60, 697-706.

44. Nichols J, Elijovich F \& Laffer CL (2012) Lack of validation of a same-day outpatient protocol for determination of salt sensitivity of blood pressure. Hypertension 59, 390-394.

45. Sassani P, Pushkin A, Gross E, et al. (2002) Functional characterization of NBC4: a new electrogenic sodium-bicarbonate cotransporter. Am J Physiol Cell Physiol 282, 408-416.

46. National Institute for Health and Care Excellence (2011) Hypertension in adults: diagnosis and management. https:// www.nice.org.uk/guidance/cg127/resources/hypertensionin-adults-diagnosis-and-management-35109454941637 (accessed October 2017)

47. Appel LJ (2017) The effects of dietary factors on blood pressure. Cardiol Clin 35, 197-212.

48. Castiglioni P, Parati G, Brambilla L, et al. (2011) Detecting sodium-sensitivity in hypertensive patients. Hypertension $\mathbf{5 7}$, 180-185.

49. Damasceno A, Caupers P, Santos A, et al. (2000) Influence of salt intake on the daytime-nighttime blood pressure variation in normotensive and hypertensive black subjects. Rev Port Cardiol 19, 315-329.

50. Brian MS, Dalpiaz A, Matthews EL, et al. (2017) Dietary sodium and nocturnal blood pressure dipping in normotensive men and women. J Hum Hypertens 31, 145-150.

51. Elijovich F, Weinberger MH, Anderson CAM, et al. (2016) Salt sensitivity of blood pressure: a scientific statement from the American Heart Association. Hypertension 68, e7-e 46.

52. Scientific Advisory Committee on Nutrition (2003) Salt and Health. London: The Stationery Office.
53. Smith JL, Estus S, Lennie TA, et al. (2016) Abstract 19630: TASR genotype is associated with adherence to dietary sodium recommendations in adults with cardiovascular disease risk factors. Circulation 134, 19630.

54. Kho M, Song YM, Lee K, et al. (2012) Genetic variants explaining sodium intake in a population with higher sodium intake level: the healthy twin study, Korea. Presented at the 62nd Annual Meeting of The American Society of Human Genetics, November 2012, San Francisco, CA.

55. Vinnikova AK, Alam RI, Malik SA, et al. (2004) $\mathrm{Na}^{+}-\mathrm{H}^{+}$ exchange activity in taste receptor cells. J Neurophysiol 91, $1297-1313$.

56. Merigo F, Benati D, Cristofoletti M, et al. (2011) Glucose transporters are expressed in taste receptor cells. J Anat $\mathbf{2 1 9}$, 243-252.

57. Felder RA, Jose PA, Xu P, et al. (2016) The renal sodium bicarbonate cotransporter NBCe2: is it a major contributor to sodium and $\mathrm{pH}$ homeostasis? Curr Hypertens Rep 18, 71.

58. Wise PM, Hansen JL, Reed DR, et al. (2007) Twin study of the heritability of recognition thresholds for sour and salty taste. Chem Senses 32, 749-754.

59. Galindo-Cuspinera V, Waeber T, Antille N, et al. (2009) Reliability of threshold and suprathreshold methods for taste phenotyping: characterization with PROP and sodium chloride. Chemosens Percept 2, 214-228.

60. Millar JA \& Lever AF (2000) Implications of pulse pressure as a predictor of cardiac risk in patients with hypertension. Hypertension 36, 907-911.

61. Stütz AM, Teran-Garcia M, Rao DC, et al. (2009) Functional identification of the promoter of SLC4A5, a gene associated with cardiovascular and metabolic phenotypes in the HERITAGE Family Study. Eur I Hum Genet 17, 1481-1489.

62. Elliott P \& Brown I (2007) Sodium Intakes Around the World. Geneva: WHO.

63. Lee H, Cho HJ, Bae E, et al. (2014) Not salt taste perception but self-reported salt eating habit predicts actual salt intake. J Korean Med Sci 29, Suppl. 2, S91-S96.

64. Sarmugam R, Worsley A \& Wang W (2013) An examination of the mediating role of salt knowledge and beliefs on the relationship between socio-demographic factors and discretionary salt use: a cross-sectional study. Int J Behav Nutr Phys Act 19, $10-25$.

65. Quader ZS, Patel S, Gillespie C, et al. (2016) Trends and determinants of discretionary salt use: National Health and Nutrition Examination Survey 2003-2012. Public Health Nutr 19, 2195-2203.

66. Freedman LS, Commins JM, Moler JE, et al. (2015) Pooled results from 5 validation studies of dietary self-report instruments using recovery biomarkers for potassium and sodium intake. Am J Epidemiol 181, 473-487.

67. Sasieni P (1997) From genotypes to genes: doubling the sample size. Biometrics 53, 1253-1261.

68. Corcoran C, Mehta C \& Senchaudhuri P (2000) Power comparisons for tests of trend in dose-response studies. Stat Med 19, 3037-3050.

69. Clarke GM, Anderson CA, Pettersson FH, et al. (2011) Basic statistical analysis in genetic case-control studies. Nat Protoc 6, 121-133. 